\title{
Generation of Multiple Focal Hole Segments Using Double-Ring Shaped Azimuthally Polarized Beam
}

\author{
K. Lalithambigai, ${ }^{1}$ R. C. Saraswathi, ${ }^{1}$ P. M. Anbarasan, ${ }^{1}$ K. B. Rajesh, ${ }^{2}$ and Z. Jaroszewicz ${ }^{3,4}$ \\ ${ }^{1}$ Department of Physics, Periyar University, Salem, Tamilnadu 636 011, India \\ ${ }^{2}$ Department of Physics, Chikkanna Government Arts College, Trippur, Tamilnadu 641 602, India \\ ${ }^{3}$ Department of Physical Optics, Institute of Applied Optics, 04894 Warsaw, Poland \\ ${ }^{4}$ National Institute of Telecommunications, 04894 Warsaw, Poland
}

Correspondence should be addressed to K. B. Rajesh; rajeskb@gmail.com

Received 17 July 2012; Revised 4 January 2013; Accepted 18 February 2013

Academic Editor: Mark D. Havey

Copyright $\odot 2013 \mathrm{~K}$. Lalithambigai et al. This is an open access article distributed under the Creative Commons Attribution License, which permits unrestricted use, distribution, and reproduction in any medium, provided the original work is properly cited.

We investigated the focusing properties of a double-ring-shaped azimuthally polarized beam tightly focused with a high NA lens and a binary phase filter. We observe that by using 3 belts and 5 belts binary phase filters novel focal patterns including splitting of focal rings and multiring focus are obtained. We also found that a suitably designed phase structure can shift the focal rings along optical axis. The author expects such investigation is worthwhile for optical manipulation and material processing technologies.

\section{Introduction}

Growing interest in the generation of three-dimensional (3D) optical beams that are dark regions in space surrounded by light is driven by wide ranging applications including dark optical traps for atoms [1], manipulation, guiding and binding of microparticles and biological cells [2], and erase beams for super-resolution fluorescence microscopy [3]. Such beams are also known as twice-closed tubular optical structure (TCTOS) [4], optical bubbles [5], and hollow dark spherical spots [6]. Optical beams with oscillating on-axis intensity due to interference resulting in $3 \mathrm{D}$ intensity voids and $3 \mathrm{D}$ optical chains were proposed recently for trapping of multiple particles along the beam propagation and for controllable particle delivery [7]. Over the past years, a variety of techniques have been proposed for generating such optical bottle beams for applications in optical tweezers and atom traps [8-15]. Recently, a subwavelength focal hole $(\sim 0.5 \lambda)$ with a quite long depth of focus $(\sim 48 \lambda)$ is achieved near the focus by tight focusing of double-ring-shaped azimuthally polarized beam with high NA lens axicon [16]. In this paper we investigate the focal properties of the tightly focused azimuthally polarized double-ring-shaped beam using 3 belts and 5 belts binary phase filters. We observed that by properly designing the binary phase filters one can achieve many novel focal patterns including splitting of focal rings and generation of multiring structures.

\section{Theoretical Work}

A schematic diagram of the suggested method is shown in Figure 1. The analysis was performed on the basis of Richards and Wolf's vectorial diffraction method [17] widely used for high-NA focusing systems at arbitrary incident polarization. In the case of the azimuthally incident polarization, adopting the cylindrical coordinates $r, z$, and $\varphi$ and the notations of [18], the electric field $E(r, \varphi, z)$ in the vicinity of the focal region can be written as

$$
\begin{aligned}
E & (r, \varphi, z) \\
& =\left[\begin{array}{c}
E_{r} \\
E_{\varphi} \\
E_{z}
\end{array}\right] \\
& =\left[\begin{array}{c}
A \int_{0}^{\alpha} \cos ^{1 / 2}(\theta) \sin (2 \cdot \theta) A(\theta) J_{1}(k r \sin \theta) e^{i k z \cos \theta} d \theta \\
2 A \int_{0}^{\alpha} \cos ^{1 / 2}(\theta) \sin (\theta) A(\theta) J_{1}(k r \sin \theta) e^{i k z \cos \theta} d \theta \\
0
\end{array}\right],
\end{aligned}
$$




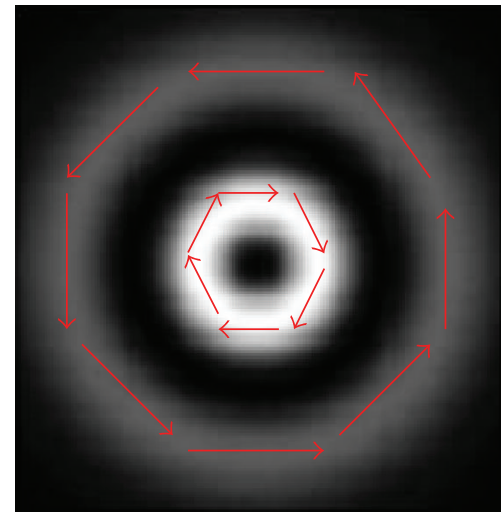

(a)

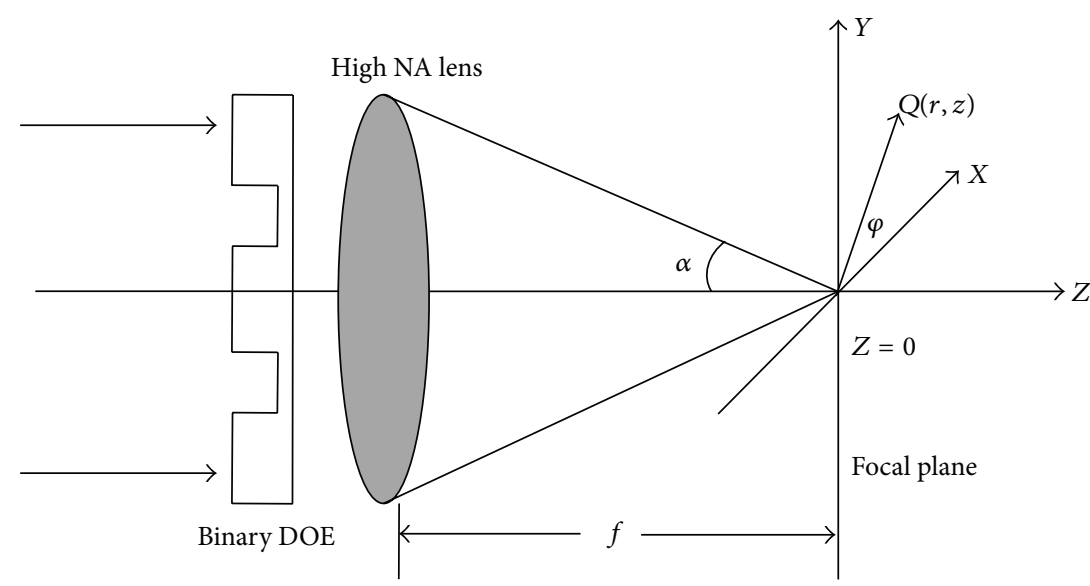

(b)

(c)

FIGURE 1: (Colour online) scheme for a double-ring-shaped azimuthally polarized beam focused by a binary phase plate with high NA lens. The red arrows in (a) shows the direction of polarization. (b) is the DOE and is placed near the lens approximately $1 / 4$ the distance between lens and the source. The point $Q(r, z)$ in (c) represents the radial and axial coordinates of the observation point at the focal plane relative to the focus point, and $\varphi$ is the azimuth angle defining the radial direction.

Here $A$ is relative amplitude, $\alpha=\arcsin \left(\mathrm{NA}_{\max } / n\right)$ is the maximum aperture angle with $\left(\mathrm{NA}_{\max } / n\right)$ is the numerical aperture, and $n$ is the index of refraction between the lens and the sample. $J_{1}(x)$ denotes the Bessel functions of first order, and the function $A(\theta)$ describes the amplitude modulation.

For illumination by a double-ring-shaped R-TEM ${ }_{11}^{*}$ beam with its waist in the pupil, this function is given by [19]

$$
A(\theta)=\beta^{2} \frac{\sin \theta}{\sin ^{2} \alpha} \exp \left[-\left(\beta \frac{\sin \theta}{\sin \alpha}\right)\right]^{2} L_{p}^{1}\left[2\left(\beta \frac{\sin \theta}{\sin \alpha}\right)^{2}\right] \text {, }
$$

where $\beta$ is the parameter that denotes the ratio of pupil diameter to the beam diameter and $L_{p}^{1}$ is the generalized Laguerre polynomial. If $p=1$, the incident azimuthally polarized beam is a double-ring-shaped azimuthally polarized beam.

\section{Analysis of Binary Phase Plate}

It is seen from (1) that an additional radial electric field component is produced after introducing the phase modulation to the input double-ring-shaped azimuthally polarized beam [20]. Owing to this radial component, the polarization near the focal plane is rather complicated and space variant. We perform the integration of (1) numerically using parameters $\lambda=1, \beta=1.3$, and $\mathrm{NA}_{\max }$ of the objective is 0.9. Here, for simplicity, we assume that the refractive index $n=1$ and $A=1$. The effect of phase modulation on the input double-ring-shaped azimuthally polarized beam is evaluated by replacing the function $A(\theta)$ by $A(\theta) T(\theta)$ where $T(\theta)$ for 3 belts binary phase plate (BPP) is given by

$$
T(\theta)= \begin{cases}1, & \text { for } 0<\mathrm{NA}<\mathrm{NA}_{1}, \mathrm{NA}_{2}<\mathrm{NA}<\mathrm{NA}_{\max }, \\ -1 & \text { for } 0, \mathrm{NA}_{1}<\mathrm{NA}<\mathrm{NA}_{2} .\end{cases}
$$

For 5-belt binary phase filter,

$$
T(\theta)= \begin{cases}1, & \text { for } 0<\mathrm{NA}<\mathrm{NA}_{1}, \mathrm{NA}_{2}<\mathrm{NA}<\mathrm{NA}_{3}, \\ & \mathrm{NA}_{4}<\mathrm{NA}<\mathrm{NA}_{\max }, \\ -1 & \text { for } \mathrm{NA}_{1}<\mathrm{NA}<\mathrm{NA}_{2}, \mathrm{NA}_{3}<\mathrm{NA}<\mathrm{NA}_{4} .\end{cases}
$$

When a double-ring-shaped azimuthally polarized beam without any phase modulation is tightly focused, a single-ring focal hole pattern is obtained and is shown in Figure 2(a). We observed that the FWHM of the generated focal hole is $0.782 \lambda$ and its corresponding focal depth is $4 \lambda$. Such a focal ring with subwavelength size can be used for stable trapping of single particle with low refractive index [21]. However, to trap two separates such particles and to manipulate them, we need to split this single-ring focal hole structure to a double ring structure. We observed that this is possible by introducing phase modulation to the input double-ringshaped azimuthally polarized beam through a 3-belt binary phase plate. We optimized the NA of the first, second, and third ring of the 3-belt binary phase plate as $\mathrm{NA}_{1}=0.1, \mathrm{NA}_{2}=$ 0.25 , and $\mathrm{NA}_{\max }=0.9$ for $\beta=1.35$ to achieve the abovementioned task, and the generated focal segment is shown in Figure 2(b). From Figure 2(b), we observed that the single focal hole is splitted into two focal holes, and the FWHM of each focal hole segment is measured as $0.468 \lambda$ and is separated by the distance of $2.8 \lambda$ between them. We observed that the residual intensity that exists between the two rings is only $35 \%$ of the total intensity measured at the focal hole. The ring focus altered by the BPP can be used to move the trapped particle from one place to another. We also observed that it is possible to increase the separation distance between the two focal rings by setting $\mathrm{NA}_{1}$ and $\mathrm{NA}_{2}$ of the $\mathrm{BPP}$ as $0.2,0.85$ and by tuning $\beta=1.5$, respectively. The corresponding focal hole segment generated is shown in Figure 2(c). From the figure we measured that the FWHM of each focal holes is $0.468 \lambda$ 


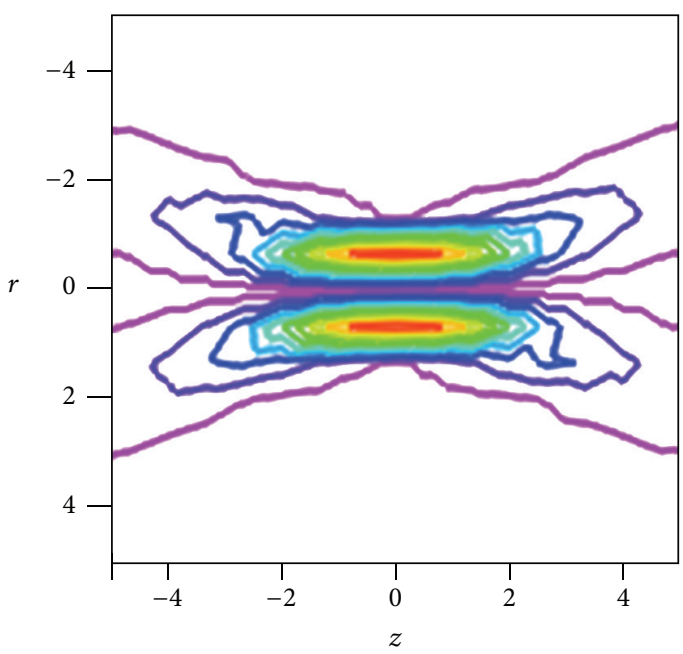

(a)

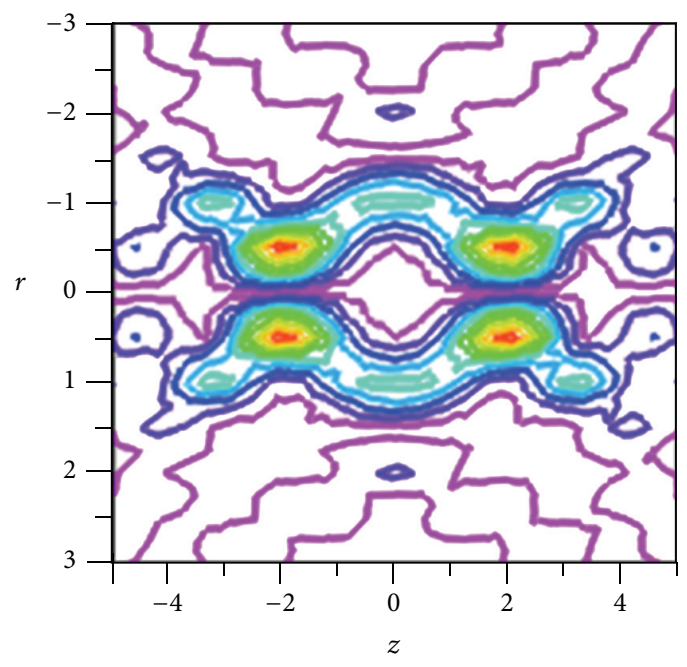

(c)

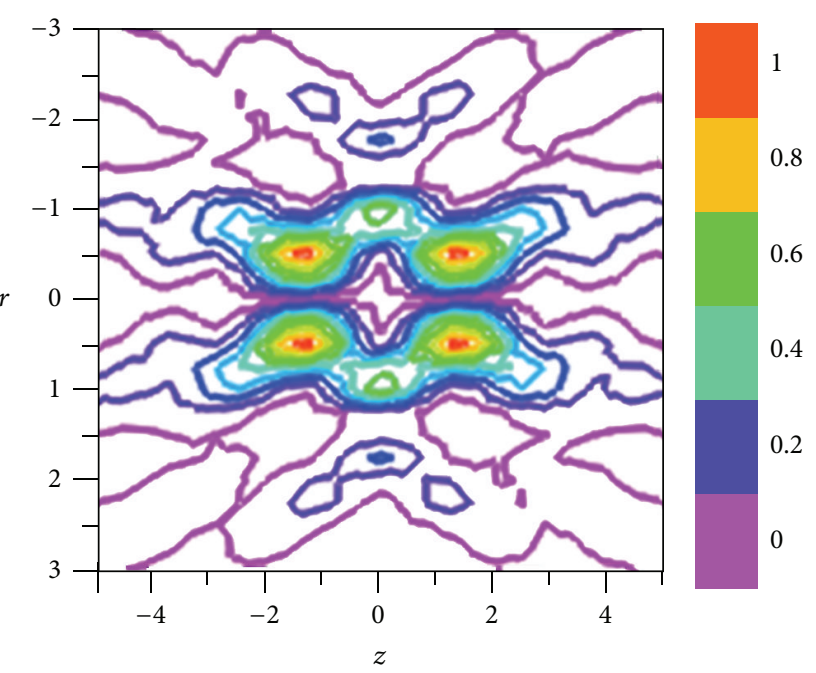

(b)

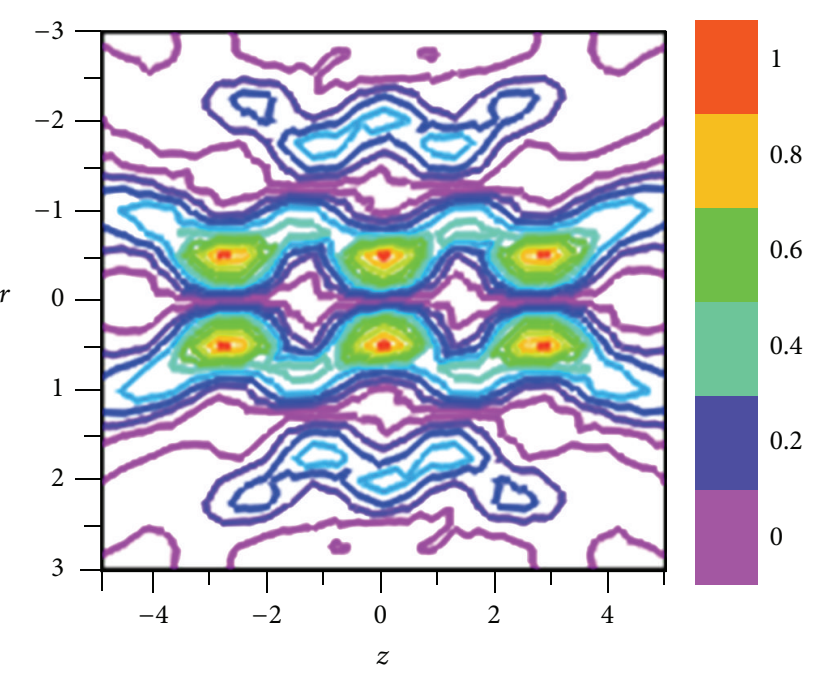

(d)

FIGURE 2: (Colour online) shows the contour plot of the total intensity distribution in the $y z$ plane near the focus for the focusing system with 3 belts BPF. (a) Single-ring focus, (b) double-ring focus, (c) double-ring focus with large separation of axial distance, and (d) triple-ring focus.

and is axially separated by the distance of $4 \lambda$ between them. We observed that the residual intensity that exists between the two rings is only $33 \%$ of the total intensity measured at the focal hole. It is also possible to achieve 3-ring focal hole segment by setting $\mathrm{NA}_{1}$ and $\mathrm{NA}_{2}$ of the $\mathrm{BPP}$ as 0.3 , 0.775 and by tuning $\beta=1.35$. The corresponding focal hole segment generated is shown in Figure 2(d). From Figure 2(d), we measured that the FWHM of each focal hole is $0.468 \lambda$, and each of them is separated by the distance of $2.8 \lambda$. We observed that the residual intensity that exists between the rings is only $31 \%$ of the total intensity measured at the focal hole. We observed that by using 5 belts BPP, it is possible to generate double ring focal segments with FWHM of $0.468 \lambda$ and are axially separated by a large distance of $9.4 \lambda$ as shown in Figure 3(a).

The set of values of NA of BPP optimized to achieve the above-mentioned task is $\mathrm{NA}_{1}=0.34, \mathrm{NA}_{2}=0.57, \mathrm{NA}_{3}=0.78$,
$\mathrm{NA}_{4}=0.85$, and $\mathrm{NA}_{\max }=0.9$ for $\beta=1.36$. From Figure 3(b) we also found that it is possible to generate four-ring patterns having almost uniform FWHM of $0.468 \lambda$ and are axially separated by the distance of $2.7 \lambda$ between them by setting $\mathrm{NA}_{1}=0.24, \mathrm{NA}_{2}=0.354, \mathrm{NA}_{3}=0.6, \mathrm{NA}_{4}=0.8$, and $\mathrm{NA}_{\max }$ $=0.9$ for $\beta=1.3$. We observed that the residual intensity that exists between the rings is only $30 \%$ of the total intensity measured at the focal hole. Thus with the help of liquid crystal-based tunable BPP, it is possible to generate tunable subwavelength focal hole segment with input double-ringshaped azimuthally polarized beam.

\section{Conclusion}

In conclusion, we show by vector diffraction theory that the tight focusing of double-ring-shaped azimuthally polarized beam with a high NA lens and tunable BPP, it is possible 


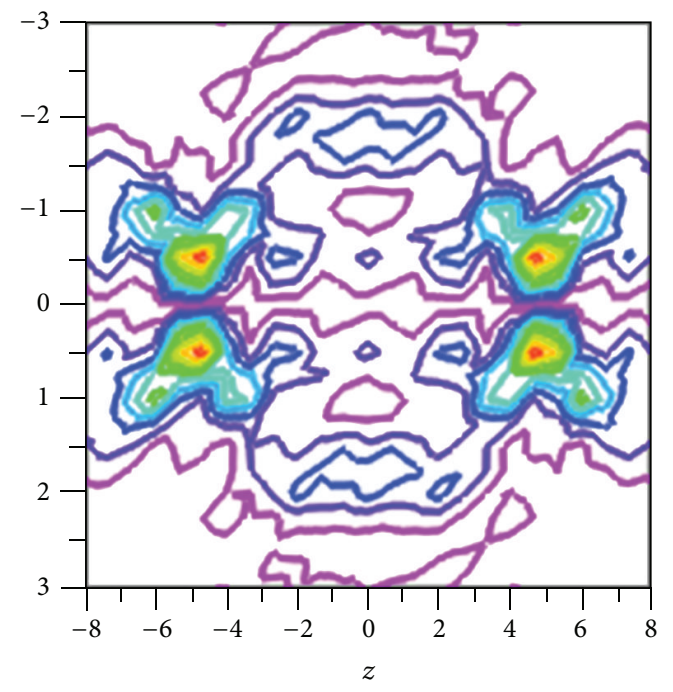

(a)

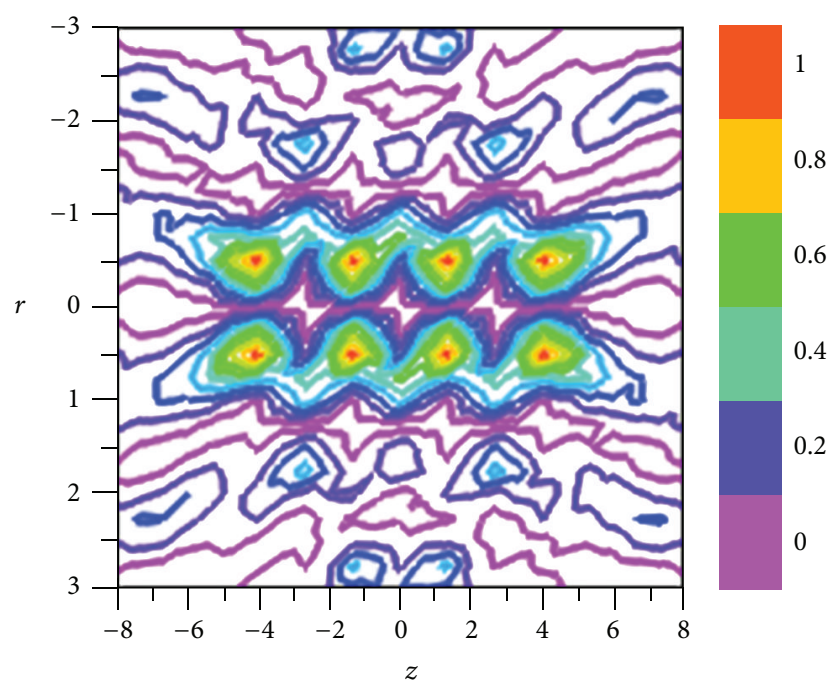

(b)

Figure 3: (Colour online) shows the contour plot of the total intensity distribution in the $y z$ plane near the focus for the focusing system with 5-belt binary phase filters. (a) Double-ring focus and (b) four-ring focus.

to generate many novel focal patterns which are potentially useful for optical trapping and manipulation of low refractive index particles.

\section{References}

[1] N. Friedman, A. Kaplan, and N. Davidson, "Dark optical traps for cold atoms," Advances in Atomic, Molecular, and Optical Physics, vol. 48, pp. 99-151, 2002.

[2] T. Cizmar, L. C. D. Romero, K. Dholakia, and D. L. Andrews, "Multiple optical trapping and binding: new routes to selfassembly," Journal of Physics B, vol. 43, Article ID 102001, 2010.

[3] T. Watanabe, Y. Iketaki, T. Omatsu, K. Yamamoto, M. Sakai, and M. Fuji, "Two point separation in super-resolution fluorescence microscope based on up-conversion fluorescence depletion technique," Optics Express, vol. 11, pp. 3271-3276, 2003.

[4] J. Garcia-Sucerquia, F. F. Medina, and G. Matteucci, "Optical tubular structures produced by diffraction of circular apertures," Optics and Lasers in Engineering, vol. 42, no. 1, pp. 61-70, 2004.

[5] W. Chen and Q. Zhan, "Three-dimensional focus shaping with cylindrical vector beams," Optics Communications, vol. 265, pp. 411-417, 2006.

[6] N. Bokor and N. Davidson, "Generation of a hollow dark spherical spot by $4 \pi$ focusing of a radially polarized LaguerreGaussian beam," Optics Letters, vol. 31, pp. 149-151, 2006.

[7] B. P. S. Ahluwalia, W. C. Cheong, X. C. Yuan et al., "Design and fabrication of a double-axicon for generation of tailorable selfimaged three-dimensional intensity voids," Optics Letters, vol. 31, no. 7, pp. 987-989, 2006.

[8] J. Arlt and M. J. Padgett, "Generation of a beam with a dark focus surrounded by regions of higher intensity: the optical bottle beam," Optics Letters, vol. 25, no. 4, pp. 191-193, 2000.

[9] B. P. S. Ahluwalia, X. C. Yuan, and S. H. Tao, "Transfer of "pure" on-axis spin angular momentum to the absorptive particle using self-imaged bottle beam optical tweezers system," Optics Express, vol. 12, no. 21, pp. 5172-5177, 2004.
[10] D. Yelin, B. E. Bouma, and G. J. Tearney, "Generating an adjustable three-dimensional dark focus," Optics Letters, vol. 29, no. 7, pp. 661-663, 2004.

[11] J. X. Pu, X. Y. Liu, and S. Nemoto, "Partially coherent bottle beams," Optics Communications, vol. 252, pp. 7-11, 2005.

[12] L. Isenhower, W. Williams, A. Dally, and M. Saffman, "Atom trapping in an interferometrically generated bottle beam trap," Optics Letters, vol. 34, no. 8, pp. 1159-1161, 2009.

[13] P. Xu, X. He, J. Wang, and M. Zhan, "Trapping a single atom in a blue detuned optical bottle beam trap," Optics Letters, vol. 35, no. 13, pp. 2164-2166, 2010.

[14] V. G. Shvedov, A. V. Rode, Y. V. Izdebskaya, A. S. Desyatnikov, W. Krolikowski, and Y. S. Kivshar, "Selective trapping of multiple particles by volume speckle field," Optics Express, vol. 18, no. 3, pp. 3137-3142, 2010.

[15] B. Tian and J. Pu, "Tight focusing of a double-ring-shaped, azimuthally polarized beam," Optics Letters, vol. 36, no. 11, pp. 2014-2016, 2011.

[16] K. Lalithambigai, P. Suresh, V. Ravi et al., "Generation of sub wavelength super-long dark channel using high NA lens axicon," Optics Letters, vol. 37, pp. 999-1001, 2012.

[17] B. Richards and E. Wolf, "Electromagnetic diffraction in optical systems-II. Structure of the image field in an aplanatic system," Proceedings of the Royal Society of London A, vol. 253, no. 1274, pp. 358-379, 1959.

[18] K. S. Youngworth and T. G. Brown, "Focusing of high numerical aperture cylindrical-vector beams," Optics Express, vol. 7, no. 2, pp. 77-87, 2000.

[19] K. B. Rajesh, N. V. Suresh, P. M. Anbarasan, K. Gokulakrishnan, and G. Mahadevan, "Tight focusing of double ring shaped radially polarized beam with high NA lens axicon," Optics \& Laser Technology, vol. 43, no. 7, pp. 1037-1040, 2011.

[20] G. H. Yuan, S. B. Wei, and X.-C. Yuan, "Nondiffracting transversally polarized beam," Optics Letters, vol. 36, pp. 3479-3481, 2011.

[21] X. Gao, S. Hu, and J. Wang, "Optical manipulation of azimuthally polarized beam altered by phase plate," IEEE Computer Society, vol. 163, pp. 657-661, 2008. 

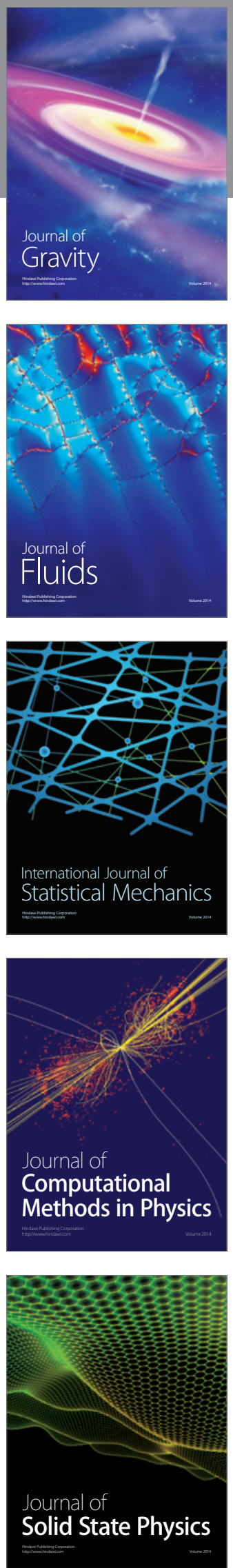

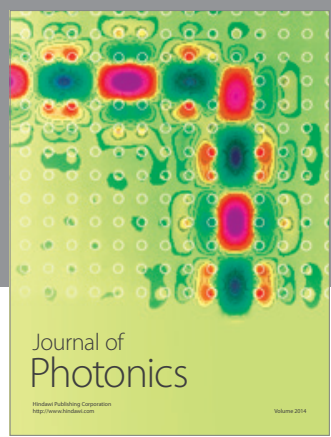

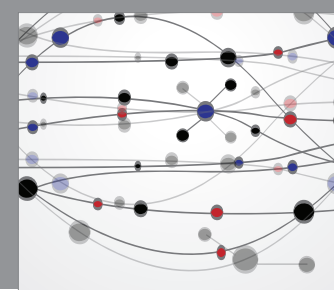

The Scientific World Journal

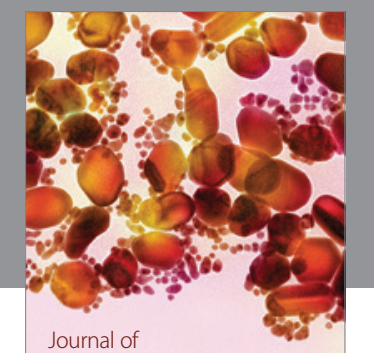

Soft Matter
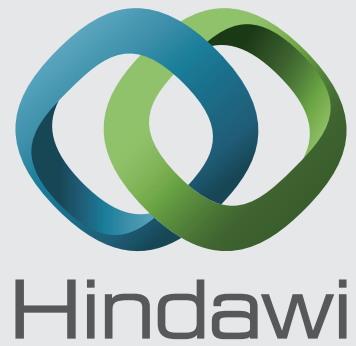

Submit your manuscripts at

http://www.hindawi.com
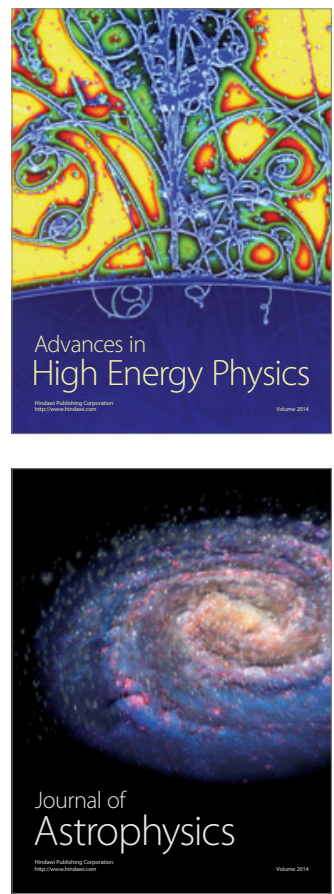
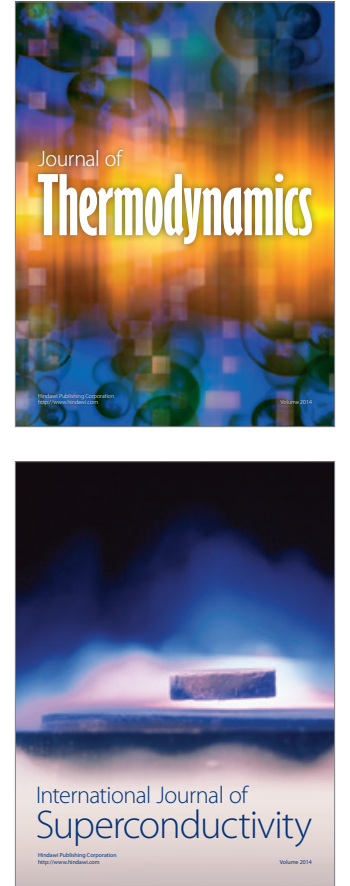
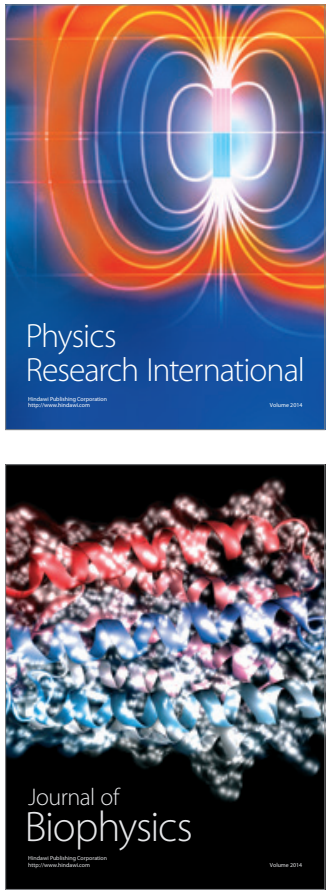
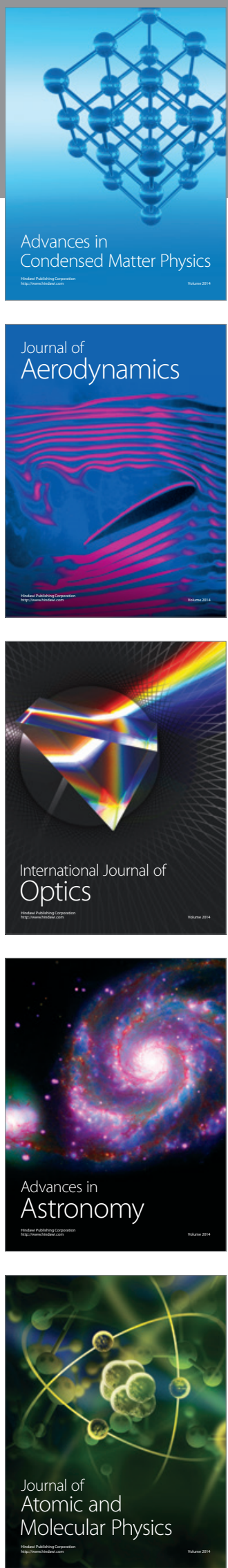